\title{
Replicability, Repeatability, and Long-term Reproducibility of Cerebellar Morphometry
}

\author{
Peter Sörös ${ }^{1,2}$ (D) Louise Wölk C Carsten Bantel $^{2,3} \cdot$ Anja Bräuer $^{2,4} \cdot$ Frank Klawonn $^{5,6} \cdot$ Karsten Witt $^{1,2}$
}

Accepted: 15 December 2020 / Published online: 9 January 2021

(C) The Author(s) 2021

\begin{abstract}
To identify robust and reproducible methods of cerebellar morphometry that can be used in future large-scale structural MRI studies, we investigated the replicability, repeatability, and long-term reproducibility of three fully automated software tools: FreeSurfer, CEREbellum Segmentation (CERES), and automatic cerebellum anatomical parcellation using U-Net with locally constrained optimization (ACAPULCO). Replicability was defined as computational replicability, determined by comparing two analyses of the same high-resolution MRI data set performed with identical analysis software and computer hardware. Repeatability was determined by comparing the analyses of two MRI scans of the same participant taken during two independent MRI sessions on the same day for the Kirby-21 study. Long-term reproducibility was assessed by analyzing two MRI scans of the same participant in the longitudinal OASIS-2 study. We determined percent difference, the image intraclass correlation coefficient, the coefficient of variation, and the intraclass correlation coefficient between two analyses. Our results show that CERES and ACAPULCO use stochastic algorithms that result in surprisingly high differences between identical analyses for ACAPULCO and small differences for CERES. Changes between two consecutive scans from the Kirby-21 study were less than $\pm 5 \%$ in most cases for FreeSurfer and CERES (i.e., demonstrating high repeatability). As expected, long-term reproducibility was lower than repeatability for all software tools. In summary, CERES is an accurate, as demonstrated before, and reproducible tool for fully automated segmentation and parcellation of the cerebellum. We conclude with recommendations for the assessment of replicability, repeatability, and long-term reproducibility in future studies on cerebellar structure.
\end{abstract}

Keywords Cerebellum $\cdot$ Segmentation $\cdot$ Parcellation $\cdot$ FreeSurfer $\cdot$ CERES $\cdot$ Reproducibility

Frank Klawonn and Karsten Witt share senior authorship.

Peter Sörös

peter.soros@gmail.com

1 Department of Neurology, Carl von Ossietzky University of Oldenburg, Heiligengeisthöfe 4, 26121 Oldenburg, Germany

2 Research Center Neurosensory Science, Carl von Ossietzky University of Oldenburg, Oldenburg, Germany

3 Anesthesiology, Critical Care, Emergency Medicine, and Pain Management, Carl von Ossietzky University of Oldenburg, Oldenburg, Germany

4 Department of Anatomy, Carl von Ossietzky University of Oldenburg, Oldenburg, Germany

5 Biostatistics, Helmholtz Centre for Infection Research, Braunschweig, Germany

6 Department of Computer Science, Ostfalia University of Applied Sciences, Wolfenbüttel, Germany

\section{Introduction}

Physiology and pathophysiology of the cerebellum have received growing attention in basic and clinical neurosciences [1-3]. Early nineteenth century neuroscientists, especially Luigi Rolando and Pierre Flourens, have established the crucial role of the cerebellum in motor control [4] and, more specifically, motor coordination [5]. More recently, the role of motor learning $[6,7]$ and the non-motor functions of the cerebellum [8] have been investigated in greater detail. The cerebellar contributions to various cognitive [9] and emotional functions [10] as well as timing [11, 12] have been acknowledged. Moreover, structural changes of the cerebellum in healthy aging [13] and neurodegenerative disease [14, 15] have been studied.

The advent of magnetic resonance imaging (MRI) has opened the door to quantitative, non-invasive investigations of cerebellar morphology. The segmentation of the cerebellum into gray and white matter and the parcellation into lobes and 
single lobules turned out to be challenging because of its tightly folded structure, consisting of numerous small folia, the equivalent of cerebral gyri. Moreover, the anatomy of the cerebellum is characterized by pronounced interindividual differences [16, 17]. Manual slice-by-slice labeling of MRIs by an expert neuroanatomist is considered the gold standard of cerebellar research [18]. Nevertheless, manual segmentation and parcellation have major disadvantages, requiring expert knowledge and being observer-dependent and time-consuming, and are not feasible in large-scale studies. To overcome the limitations of manual identification of cerebellar structures, several fully automated methods for cerebellar morphometry have been developed and made publicly available (for a review, see Carass et al. [19]). The results of various methods have been compared with manually labeled adult and pediatric cerebellar data sets [19]. In this comparison, an improved version of the patch-based multiatlas segmentation tool CERES (CEREbellum Segmentation) [20] exhibited highest accuracy and outperformed established methods, such as the MATLAB toolbox SUIT (Spatially Unbiased Infratentorial Template) $[16,21]$. While the accuracy of CERES and other methods have been established, the reproducibility of fully automated cerebellar morphometry has not been determined so far.

In the present study, we investigate the replicability, repeatability, and long-term reproducibility of cerebellar morphometry using three independent MRI data sets and three software packages based on different computational approaches. The definitions of replicability, repeatability, and reproducibility follow the suggestions by Nichols et al. [22]. Replicability is defined as computational or analysis replicability, determined by comparing two analyses of the same MRI data set performed with identical analysis software and computer hardware. Repeatability is determined by comparing the analyses of two MRI scans of the same participant taken during two independent MRI sessions on the same day. Long-term reproducibility, finally, is assessed by analyzing two MRI scans of the same participant in a longitudinal study. We decided to test the following three software packages: (1) FreeSurfer, an established and widely used approach of subcortical segmentation, based on a probabilistic atlas, which performs cerebellar segmentation, but not parcellation [23]; (2) CERES, a recent segmentation and parcellation method based on a multiatlas label fusion technique [20], the most accurate software tool in the comparison by Carass et al. [19]; and (3) automatic cerebellum anatomical parcellation using U-Net with locally constrained optimization (ACAPULCO), a very recent and promising parcellation approach based on convolutional neural networks [24], not included in the comparison by Carass et al. [19]. In a separate paper, the developers of ACAPULCO demonstrated comparable accuracy of their software relative to CERES for adult data and even superior accuracy in several regions for pediatric data [24].
The ultimate aim of this study is to identify robust and reproducible methods of fully automated cerebellar morphometry that can be used in MRI studies with large sample sizes.

\section{Participants and Methods}

\section{MRI data}

For this study, three independent data sets of T1-weighted MRIs of the entire brain have been analyzed with three different fully automated software packages: FreeSurfer Version 7.1.0 [23], CERES [20], and ACAPULCO [24].

\section{Replicability: ChroPain2 Study}

To investigate the analysis replicability of cerebellar morphometry, we performed two separate, but identical analyses of high-resolution structural MRIs of 23 healthy individuals (17 women, 6 men) who served as control participants for the ChroPain2 study. Inclusion and exclusion criteria have been published previously [25]. Mean age \pm standard deviation was $51 \pm 10$ years (minimum: 30 years, maximum: 66 years). All participants provided written informed consent for participation in this study. The study was approved by the Medical Research Ethics Board, University of Oldenburg, Germany (2017-059) and was preregistered with the German Clinical Trials Register (DRKS00012791) ${ }^{1}$.

MR images of the entire brain were acquired in the Neuroimaging Unit, School of Medicine and Health Sciences, University of Oldenburg ${ }^{2}$, on a research-only Siemens MAGNETOM Prisma whole-body scanner (Siemens, Erlangen, Germany) at 3 Tesla with a 64-channel head/neck receive-array coil. A 3-dimensional high-resolution and high-contrast T1-weighted magnetization-prepared rapid gradient echo (MPRAGE) sequence was used [26]. Imaging parameters were as follows: TR (repetition time; between two successive inversion pulses): $2000 \mathrm{~ms}$, TE (echo time): 2.07 $\mathrm{ms}$, TI (inversion time): $952 \mathrm{~ms}$, flip angle: $9^{\circ}$, isotropic voxel size: $0.75 \times 0.75 \times 0.75 \mathrm{~mm}^{3}, 224$ sagittal slices, k-space interpolation-based in-plane acceleration (GRAPPA) with an acceleration factor of 2 [27], and time of acquisition: 6:16 $\mathrm{min}$. Siemens' prescan normalization filter was used for online compensation of regional signal inhomogeneities.

\section{Repeatability: Kirby-21 Study}

To investigate repeatability of cerebellar morphometry, we analyzed data from the Kirby-21 multi-modal MRI reproducibility study [28], performed at the F.M. Kirby Research

\footnotetext{
${ }^{1}$ www.drks.de

2 uol.de/en/medicine/biomedicum/neuroimaging-unit
} 
Center for Functional Brain Imaging, Kennedy Krieger Institute, Baltimore, MD, USA. For this study, each participant received two identical MRI examinations on the same day, each consisting of several sequences, including a T1weighted MPRAGE sequence. After the first examination, participants left the scanner room for a short break and were then repositioned and scanned with the identical imaging protocol a second time. The time interval between the two T1weighted images was approximately $1 \mathrm{~h}$. MRIs were acquired from 21 individuals (10 women, 11 men) with no history of neurological disorders. Mean age \pm standard deviation was 32 \pm 9 years (minimum: 22 years, maximum: 61 years). (For a detailed description of the entire study, see [28].) The data set is publicly available for download ${ }^{3}$ and has been used in several studies on the reproducibility of MRI analyses (e.g., [29, 30]).

MR images of the entire brain were acquired at 3 Tesla using a Philips Achieva MR scanner (Philips Healthcare, Best, The Netherlands) with an 8-channel receive-array head coil.

Imaging parameters for the MPRAGE sequence were as follows: TR (between two successive gradient echoes): 6.7 ms, TE: $3.1 \mathrm{~ms}$, TI: $842 \mathrm{~ms}$, flip angle: $8^{\circ}$, voxel size: $1 \times 1$ $\times 1.2 \mathrm{~mm}^{3}$, image domain-based in-plane acceleration (SENSE) with an acceleration factor of 2, and duration: 5:56 $\min$.

\section{Long-term Reproducibility: OASIS-2 Study}

To investigate long-term reproducibility of cerebellar morphometry, we performed analyses of MR images acquired for the Open Access Series of Imaging Studies (OASIS-2) [31], performed at the Washington University School of Medicine, St. Louis, MO, USA. The OASIS-2 study comprises longitudinal MR examinations of patients with Alzheimer's disease and healthy controls. For the present study of cerebellar morphometry, we analyzed the data of 72 individuals (50 women, 22 men) who remained cognitively unimpaired throughout the study, as demonstrated by a Clinical Dementia Rating (CDR) score of 0 [32]. Mean age at inclusion $\pm \mathrm{SD}$ was $75 \pm 8$ years (minimum: 60 years, maximum: 93 years). For the OASIS- 2 study, participants received 2-5 MRI examinations months or years apart; each MRI examination consisted of 3-4 T1-weighted MRI scans. For the present study, we only considered the first two MRI examinations of each participant. If more than one MRI scan was available for one examination, we chose the first one. The mean interval $\pm \mathrm{SD}$ between the two MRIs was $738 \pm 249$ days (minimum: 182, maximum: 1510 days). All MRIs were obtained with the same scanner with identical pulse sequences. (For a detailed description of the study and the

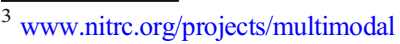

CDR scale, see Marcus et al. [31].) OASIS-2 data sets are publicly available for download ${ }^{4}$.

MR images of the entire brain were acquired on a Siemens Vision whole-body scanner (Siemens, Erlangen, Germany) at 1.5 Tesla. Imaging parameters for the MPRAGE sequence were TR (between two successive gradient echoes): $9.7 \mathrm{~ms}$, TE: $4 \mathrm{~ms}$, TI $20 \mathrm{~ms}$, flip angle: $10^{\circ}$, voxel size: $1 \times 1 \times 1.25$ $\mathrm{mm}^{3}$, and 128 sagittal slices.

\section{Data Analysis}

FreeSurfer and ACAPULCO analyses were performed on the high-performance computer cluster $\mathrm{CARL}^{5}$ at the University of Oldenburg, Germany, running Red Hat Enterprise Linux. CERES was run through the online MRI Brain Volumetry System volBrain [33]. CERES can only be used through the volBrain website and was not available for installation on our computer cluster. All analyses were done fully automated. Manual editing of output images was not performed, because the aim of this study was to assess reproducibility of cerebellar morphometry for future use in large-scale data sets.

\section{FreeSurfer}

For automated analysis of subcortical structures, including the cerebellum, the FreeSurfer Version 7.1.0 RRID:SCR_001847 image analysis suite was used, which is freely available for download online ${ }^{6}$ [36]. Processing was done with the recon-all -all command. For the ChroPain2 and the Kirby-21 data sets, the $-3 T$ and mprage flags were used. For the OASIS2 data sets, the mprage flag was used. Processing started with automated transformation to Talairach space, followed by intensity normalization of the output images and removal of non-brain tissue using a hybrid approach that combines watershed algorithms and deformable surface models [37]. During segmentation, a neuroanatomical label is assigned to all voxels of the T1-weighted MRI based on a probabilistic atlas, derived from a manually labeled training set [23], using a Bayesian approach. Details of atlas construction, registration of the probabilistic atlas to the individual MRI, and segmentation based on the assumption that spatial distribution of labels can be approximated by an anisotropic nonstationary Markov random field are given by Fischl et al. [23]. FreeSurfer reports the volumes of the left and right cerebellar cortex and the left and right cerebellar white matter (Fig. 1). Parallelization was not used; all processes were run on a single computer core of a high-performance computer cluster. Processing of the first MRI of the first MR examination of participant OAS2_0095

\footnotetext{
$\overline{4_{\text {www.oasis-brains.org }}}$

${ }^{5}$ uol.de/en/school5/sc/high-perfomance-computing/hpc-facilities/carl

${ }^{6}$ https://surfer.nmr.mgh.harvard.edu/fswiki/rel7download
} 


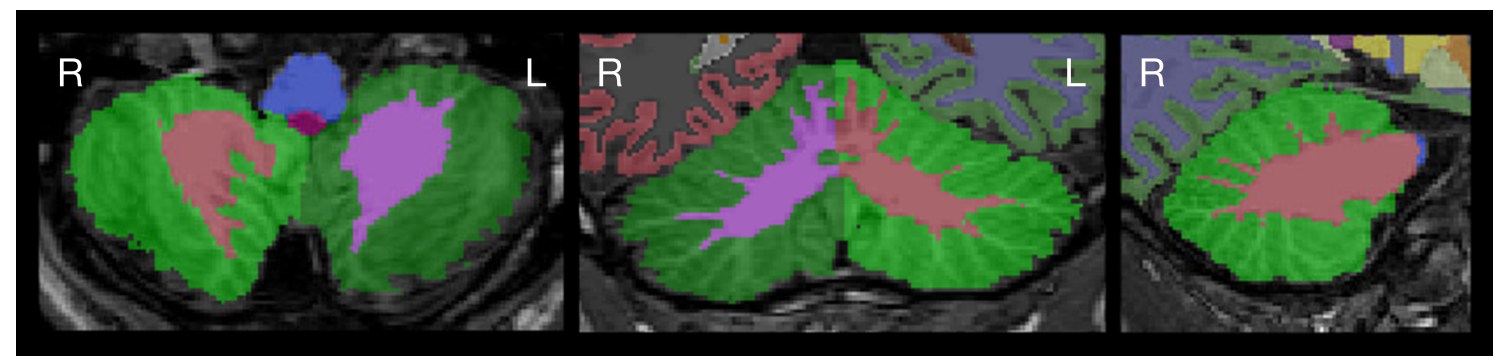

Fig. 1 Cerebellar segmentation as determined by FreeSurfer. Images were created with FSLeyes. The left image shows a horizontal, the middle image a coronal, and the right image a sagittal section of the

failed due to an error during topology correction (with and without the -mprage flag). We analyzed the second MRI of the first examination instead; processing finished without error.

\section{CERES}

CERES is an automated pipeline for cerebellar segmentation and parcellation [20] and is part of the volBrain Automated MRI Brain Volumetry System [33]. In brief, CERES receives an anonymized T1-weighted MRI brain volume in NIfTI format through the volBrain website ${ }^{7}$, performs image preprocessing, and labels cerebellar voxels based on Optimized PatchMatch Label fusion [38]. Preprocessing includes (1) denoising [39], (2) bias field correction using the N4 algorithm [40], (3) linear registration to the MNI152 standard space template using Advanced Normalization Tools (ANTs) [41, 42], (4) cropping of the cerebellum area, (5) nonlinear registration to the cropped MNI152 template using ANTs [41, 42], and (6) local intensity normalization. Labeling of cerebellar voxels was performed with non-local patchbased label fusion, a multiatlas segmentation technique combining segmentations from multiple reference atlases, initially developed for hippocampal segmentation [43, 44]. The atlases were created based on manually segmented high-resolution MR images from 5 healthy volunteers ( 3 women, 2 men, aged 29-57 years) [18], available for download ${ }^{8}$. CERES determines the entire volume, cortical thickness, and gray matter volume of all regions listed in Table 1, separately for the left and right side of the cerebellum (Fig. 2). Of note, we have used the publicly available first version of CERES. All analysis steps have been determined by the developers; changes of analysis methods or parameters are not possible. In the study on accuracy of cerebellar morphometry performed by Carass et al. [19], an improved version (CERES2) was tested, which employs an improved intensity normalization method and a systematic error correction step; CERES2 has not been released for public use so far.

\footnotetext{
$\overline{7}$ volbrain.upv.es

${ }^{8}$ cobralab.ca/atlases/
}

cerebellum. Images are in radiological convention (the left side of the cerebellum is on the right side of the image). Cerebellar cortex is displayed in green color

\section{ACAPULCO}

Han et al. [24] developed a method using convolutional neural networks for cerebellar parcellation (ACAPULCO). ACAPULCO processes T1-weighted images of the brain in NIfTI format, preferentially acquired with an MPRAGE sequence. A Singularity image of this software is publicly available ${ }^{9}$. We ran this image on University of Oldenburg's HPC cluster using Singularity 2.6.

As suggested by the developers, all images were first cropped with the robustfov command provided by FSL ${ }^{10}$ to remove the lower head and neck in MRIs with large field-ofview. Processing within ACAPULCO included (1) estimation of a brain mask using Robust Brain Extraction (ROBEX) ${ }^{11}$ [45] for subsequent bias field correction, (2) bias field correction using the N4 algorithm [40], (3) linear registration to MNI space using the 1-mm isotropic ICBM 2009c nonlinear symmetric template ${ }^{12}$ using ANTs [41, 42], (4) parcellation of the cerebellum as described by Han et al. [24], and (5) transformation of the parcellation into original space using ANTs with the MultiLabel interpolation. For cerebellar parcellation, ACAPULCO employs two three-dimensional convolutional neural networks. First, a locating network is used to predict a bounding box around the cerebellum. Second, a parcellating network is used to parcellate the cerebellum using the entire region within the bounding box [24]. ACAPULCO employs the TensorFlow software library for Python ${ }^{13}$ and the GNU Parallel tool [46]. The cerebellar regions identified by ACAPULCO are summarized in Table 1; ACAPULCO reports the entire volume of the left and right lobules and the vermis regions in the midline (Fig. 3). Of note, all analysis steps have been determined by the developers; changes of analysis methods or parameters are not possible.

Unexpectedly, we found large differences between the first and second analysis of the high-resolution ChroPain2 data set using ACAPULCO. To investigate

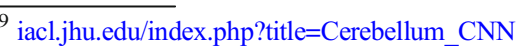

${ }^{10}$ fsl.fmrib.ox.ac.uk/fsl/fslwiki/InitialProcessing

${ }^{11}$ www.nitrc.org/projects/robex/

12 www.bic.mni.mcgill.ca/ServicesAtlases/ICBM152NLin2009

13 www.tensorflow.org
} 
Table 1 Cerebellar regions parcellated in CERES and ACAPULCO. CERES determines the entire volume $\left(\mathrm{cm}^{3}\right)$, the mean cortical thickness $(\mathrm{mm})$, and the gray matter volume $\left(\mathrm{cm}^{3}\right)$ of each region.

ACAPULCO determines the

volume $\left(\mathrm{mm}^{3}\right)$ of each region.

CERES and ACAPULCO make use of the cerebellar nomenclature proposed by Schmahmann et al. [34]. In addition, the traditional names of vermical regions according to the Terminologia Anatomica [35] are listed. The less common names of hemispheric regions were omitted

\begin{tabular}{|c|c|c|c|}
\hline \multirow[t]{2}{*}{ CERES } & \multicolumn{2}{|l|}{ ACAPULCO } & \multirow{2}{*}{$\begin{array}{l}\text { Terminologia Anatomica } \\
\text { Vermis }\end{array}$} \\
\hline & Vermis & Hemisphere & \\
\hline \multicolumn{4}{|l|}{ Anterior lobe } \\
\hline \multirow[t]{2}{*}{ Lobules I-II } & & Lobules I-III & Lobulus I: lingula \\
\hline & & & Lobulus II: centralis \\
\hline Lobule III & & & Lobulus III: centralis \\
\hline Lobule IV & & Lobule IV & Lobulus IV: culmen \\
\hline Lobule V & & Lobule V & Lobulus V: culmen \\
\hline \multicolumn{4}{|l|}{ Posterior lobe } \\
\hline Lobule VI & Vermis VI & Lobule VI & Lobulus VI: declive \\
\hline Crus I & & Lobule VIIAf & Lobulus VIIA: folium vermis \\
\hline Crus II & Vermis VII & Lobule VIIAt & Lobulus VIIA: folium vermis \\
\hline Lobule VIIB & & Lobule VIIB & Lobulus VIIB: tuber \\
\hline Lobule VIIIA & Vermis VIII & Lobule VIIIA & Lobulus VIIIA: pyramis \\
\hline Lobule VIIIB & Vermis VIII & Lobule VIIIB & Lobulus VIIIB: pyramis \\
\hline Lobule IX & Vermis IX & Lobule IX & Lobulus IX: uvula \\
\hline \multicolumn{4}{|c|}{ Flocculonodular lobe } \\
\hline Lobule X & Vermis X & Lobule X & Lobulus X: nodulus \\
\hline
\end{tabular}

ACAPULCO's analysis replicability with a different data set using larger voxel sizes, we performed another two separate analyses of the T1-weighted MRIs available in the Kirby-21 study using Singularity 3.4 (in the meantime, Singularity 2.6 had been deleted from the cluster). For scan KKI2009-33, the locating network of ACAPULCO predicted an incorrect bounding box in one of these analyses, placing it well above the cerebellum, leading to erroneous results of the parcellating network. This scan was excluded from the assessment of analysis replicability in the Kirby-21 study (Fig. 4c).

\section{Statistical Analysis}

For further data analyses, we calculated the percent difference between the first and second analysis of one MRI (ChroPain2

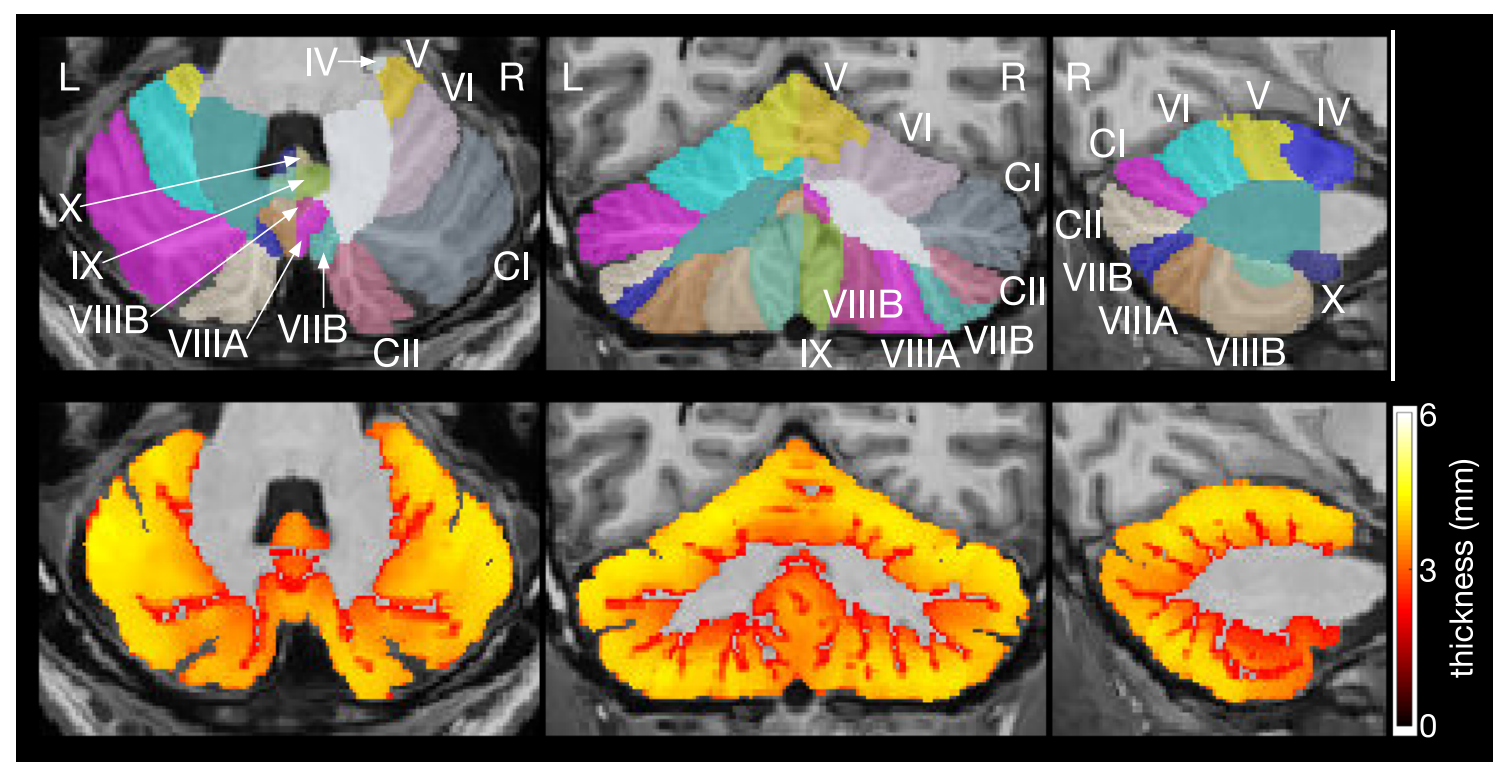

Fig. 2 Cerebellar parcellation (upper row) and thickness of cerebellar cortex (lower row) as determined by CERES. The left images show horizontal, the middle images coronal, and the right images sagittal sections of the cerebellum. Images were produced by CERES and are in neurological convention (the left side of the cerebellum is on the left side of the image). The roman numerals of the cerebellar lobules were added. CI denotes Crus I; CII, Crus II 


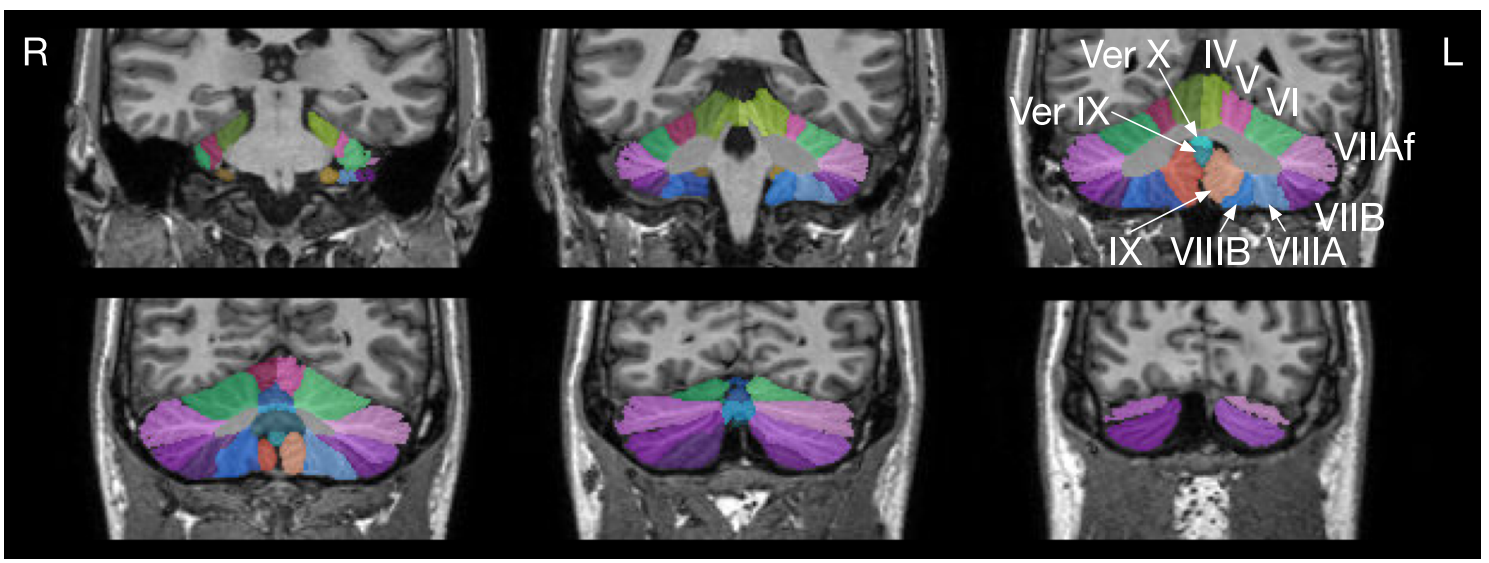

Fig. 3 Cerebellar parcellation as determined by ACAPULCO. The upper left image is the most anterior coronal section. Images were produced by ACAPULCO. The upper part of each image was cropped and the roman

study) or the first and second MRI (Kirby-21 and OASIS-2 studies), and determined the coefficient of variation (CV) and the intraclass correlation coefficient (ICC) for each cerebellar region. We also computed the image intraclass correlation coefficient (I2C2) [47] for cerebellar parcellations obtained by CERES.

\section{a FreeSurfer: hemispheric volume}

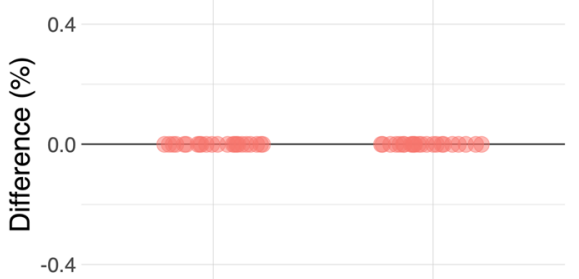

R gray matter R white matter

\section{d CERES: lobular volume}

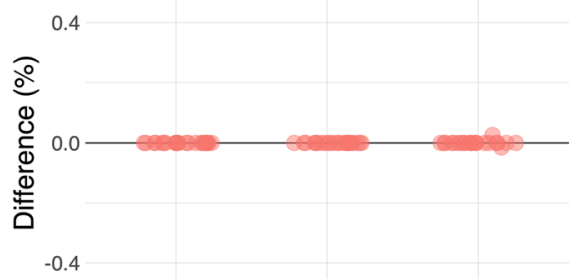

R lobule V R lobule VI R lobule VIIIA

$-20$

R lobule V R lobule VI R lobule VIIIA

e CERES: cortical thickness

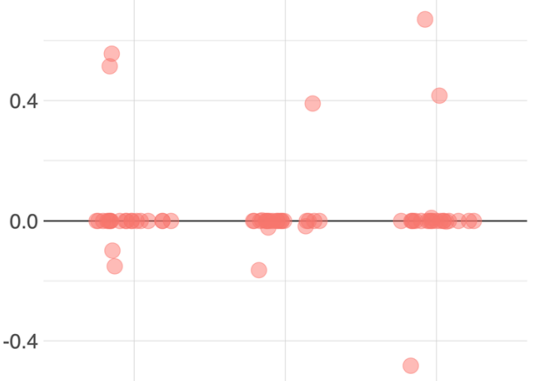

R lobule V

R lobule VI numerals of the cerebellar lobules were added. Ver IX denotes Vermis IX I; Ver X, Vermis X. Images are in radiological convention (left side of the cerebellum is on the right side of the image)

\section{Coefficient of Variation}

The coefficient of variation (CV) describes the level of variability within a sample independently of the absolute values of the observations. To calculate the $\mathrm{CV}$, the standard deviation across all measurements of one parameter (including the

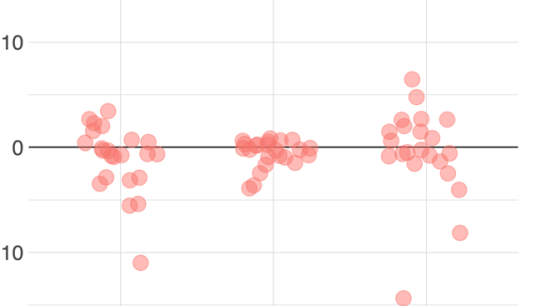

b ACAPULCO: lobular volume (ChroPain) 20

$-10$
C ACAPULCO: lobular volume (Kirby-21)

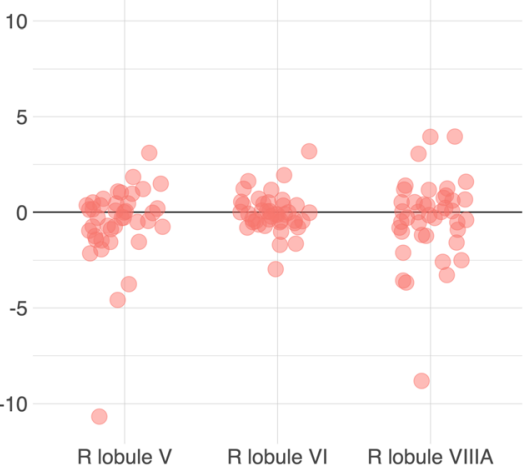

f CERES: gray matter volume

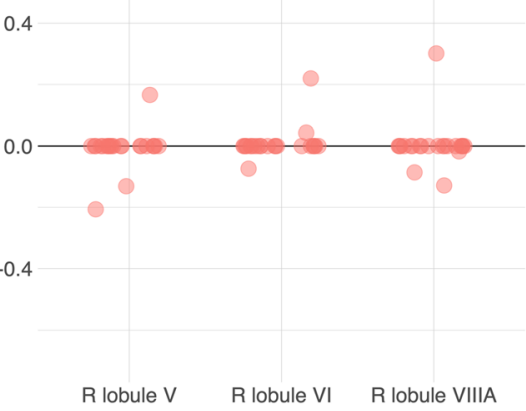

Fig. 4 Analysis replicability of cerebellar morphometry in the ChroPain2 study using FreeSurfer (a), ACAPULCO (b), and CERES (d, e, f). Analysis replicability was also assessed with all T1-weighted MRIs of the Kirby-21 study using ACAPULCO (c). The graphs show percent difference between the first and second analysis of the same data set for right gray and white matter (FreeSurfer) and the right lobules V, VI, and VIIIA (ACAPULCO, CERES). Note: the scales of the $y$ axes differ across graphs 
results of the first and second analysis of one MRI or the analyses of the first and second MRI) was divided by the (absolute) mean across all measurements and expressed as percentage. In addition, the lower and upper $95 \%$ confidence intervals were estimated using $\mathrm{R}$ for Windows [48].

\section{Intraclass Correlation Coefficient}

The intraclass correlation coefficient (ICC) is a measure of within-subject variability relative to between-subject variability. ICC estimates and their lower and upper $95 \%$ confidence intervals were calculated using the R package ssych $^{14}$ and the function ICC [49]. Following the suggestions of Liljequist et al. [50], we first calculated all three single-measurement ICCs $[51,52]$. The results of all three formulas were very similar, indicating the absence of bias (systematic error). Hence, we report the one-way random effects, absolute agreement, single-measurement ICC according to McGraw and Wong [52] or the ICC (1.1) according to Shrout and Fleiss [51]. ICC confidence intervals indicate poor reliability $(<0.5)$, moderate reliability $(0.5-0.75)$, good reliability $(0.75-0.9)$, or excellent reliability $(>0.9)[53]$.

\section{Image Intraclass Correlation Coefficient}

The image intraclass correlation coefficient $\mathrm{I} 2 \mathrm{C} 2$ has been developed as a global measure of reliability for imaging data ${ }^{15}$ [47]. The I2C2 was calculated for all cerebellar parcellations obtained by CERES for the Kirby- 21 and OASIS-2 data sets using the I2C2 package version 0.2.4 [47] for Neuroconductor [54]. First, all parcellated images created by CERES were split into 24 image files containing one parcellation only (labels 112 for the left cerebellum, labels 101-112 for the right cerebellum). Then, .nii files were imported into $\mathrm{R}$ using the readnii function of the neurobase package for Neuroconductor. Finally, the $\mathrm{I} 2 \mathrm{C} 2$ and the nonparametrically bootstrapped $95 \%$ confidence interval of the $\mathrm{I} 2 \mathrm{C} 2$ (with 1000 repetitions) between the first and second image of each participant were estimated.

\section{Results}

In this section, we will visualize results of cerebellar morphometry for the right lobules V, VI, and VIIIA obtained by CERES and ACAPULCO (Figs. 4, 5, and 6). These lobules were chosen because of their critical role in motor and nonmotor functions of the cerebellum. According to an activation likelihood estimate meta-analysis of neuroimaging studies [55], (1) the right lobule $\mathrm{V}$ is associated with motor and

\footnotetext{
${ }^{14}$ https://cran.r-project.org/web/packages/psych/index.html

15 https://neuroconductor.org/package/I2C2
}

somatosensory processing, (2) the right lobule VI is associated with motor, spatial, language, working memory, and emotional processing, and (3) the right lobule VIIIA is associated with motor and working memory processing.

We will also present the coefficient of variation (CV) and the intraclass correlation coefficient (ICC) for the results of all analyses with FreeSurfer, CERES, and ACAPULCO (Tables 3 and 4). For CERES parcellations, we will also provide the image intraclass correlation coefficients (I2C2) (Table 2). Supplementary data are available at the Open Science Framework (OSF) ${ }^{16}$.

\section{Visual Inspection}

All fully automated analyses resulted in anatomically broadly correct segmentations and parcellations except one FreeSurfer analysis (analysis failure) and one ACAPULCO analysis (incorrect placement of the bounding box localizing the cerebellum). In several FreeSurfer analyses, voxels containing dura and surrounding non-brain tissue were mislabeled as cerebellum, in particular in the midline. In single ACAPULCO analyses, the parcellation algorithm mislabeled voxels located in the neck as cerebellum, even after postprocessing (e.g., the second examination of participant OAS2_0013 in the OASIS-2 study). Visual inspection of all CERES analyses did not reveal remarkable inaccuracies.

\section{Replicability}

Using FreeSurfer with data from the ChroPain2 study, two identical analyses of the same T1-weighted image provided identical results for all participants regarding gray and white matter volumes.

Using CERES with data from the ChroPain2 study, two identical analyses provided identical results for lobular volumes, cortical thickness, and gray matter volumes in most participants (Fig. 4d, e, f for right lobules V, VI, VIIIA). For lobular volumes, differences for all regions were smaller than $\pm 0.1 \%$. For cortical thickness, maximum differences were found in the left lobules I-II $(-4.8-3.7 \%)$. Maximum differences in gray matter volume were also found in the left lobules I-II (- 5.8-11.8\%, data not shown).

Using ACAPULCO with data from the ChroPain2 study, two identical analyses provided different results for all regions. Differences were larger than those found with CERES (Fig. 4b). Differences were between $-36.6 \%$ (right lobule VIIIB) and $20.6 \%$ (right lobule IX). To confirm these results, the analysis replicability of ACAPULCO was also assessed with all T1-weighted images of the Kirby-21 study (Fig. 4c). For this data set, differences were between $-11.5 \%$ (vermis X) and 19.4\% (left lobule VIIIB, data not shown).

\footnotetext{
${ }^{16}$ https://osf.io/n8y5h/
} 


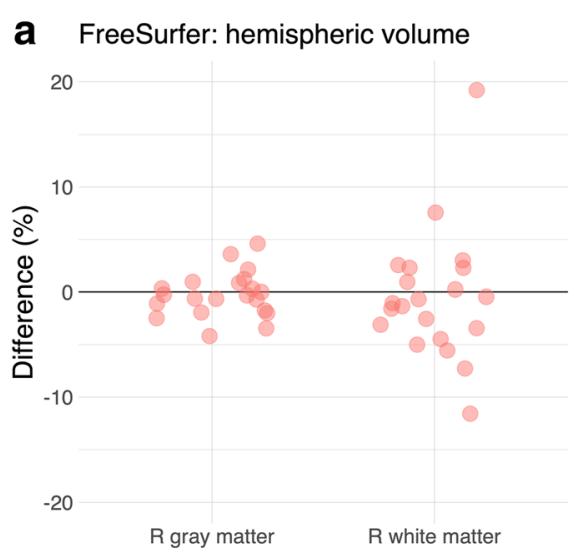

\section{CERES: lobular volume}

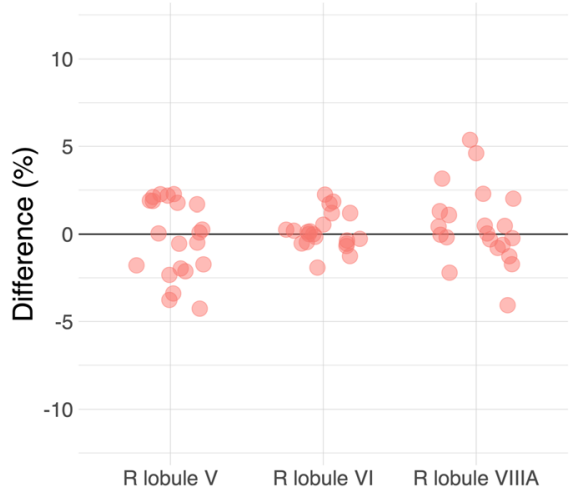

b ACAPULCO: lobular volume

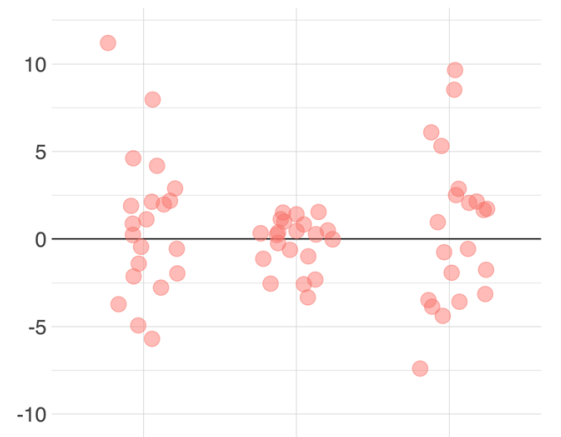

R lobule V R lobule VI R lobule VIIIA

d CERES: cortical thickness

e CERES: gray matter volume

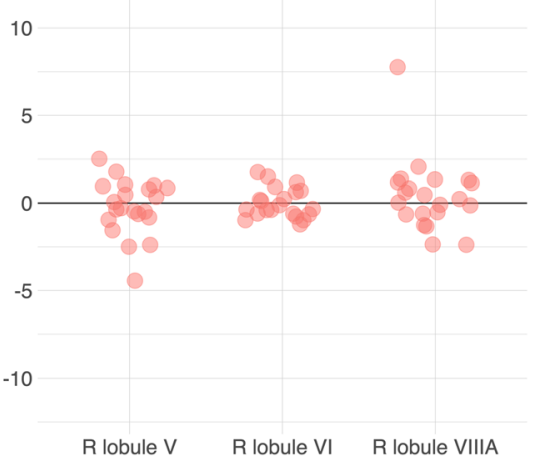

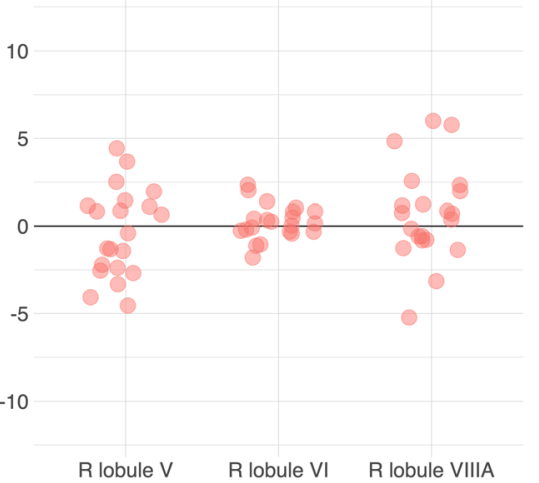

Fig. 5 Repeatability of cerebellar morphometry in the Kirby-21 study. The graphs show percent difference between the first and second MRI (a) and the right lobules V, VI, and VIIIA using ACAPULCO (b) and CERES (c, d, e). Note: the scales of the $y$ axes differ across graphs

\section{Repeatability}

Comparing the FreeSurfer results of the first and second T1weighted MRI in the Kirby-21 study, differences in gray matter volumes were below $\pm 5 \%$ (Fig. 5a). Differences in white matter volumes were higher, between -12.1 and $19.2 \%$. With CERES, differences in lobular volumes, cortical thickness, and gray matter volume were below $\pm 5 \%$ in most cases (Fig. 5c, d, e). In some cases, differences were considerably higher, in particular for the small lobules I-II. With ACAPULCO, differences in lobular volumes were also below $\pm 5 \%$ in most cases (Fig. 5b). Maximum differences were between $-20 \%$ (left lobule VIIB) and 35.1\% (left lobule VIIIA, data not shown).

The image intraclass correlation coefficients (I2C2) for repeatability using the CERES parcellations are presented in Table 2. The coefficients of variation and the intraclass correlation coefficients for repeatability are presented in Table 3. Most lower 95\% confidence intervals suggest good or even excellent repeatability.

\section{Long-term Reproducibility}

Comparing the FreeSurfer results of the first and second T1weighted MRI in the OASIS2 study, most differences in gray matter volume were below $\pm 5 \%$ (Fig. 6a). Maximum differences in gray matter volume were between -12.3 and $9.7 \%$, in white matter volume between -15.6 and $25.5 \%$. With CERES, most differences for lobular volumes, cortical thickness, and gray matter volumes were below $\pm 10 \%$, many even below $\pm 5 \%$ (Fig. 6c, d, e). Maximum differences for lobular volumes $(-60.1 \%, 167.5 \%)$, cortical thickness $(-54.4 \%$, $190.8 \%)$, and for gray matter volumes $(-55.3 \%, 139.5 \%)$ were considerably higher. With ACAPULCO, differences were also below $\pm 10 \%$ in the majority of cases, many even below $\pm 5 \%$ (Fig. 6b). Maximum differences were between 96.4 and $180.9 \%$. The image intraclass correlation coefficients using the CERES parcellations suggest moderate reproducibility (data not shown). The coefficients of variation and the intraclass correlation coefficients for reproducibility are presented in Table 4. 
a FreeSurfer: hemispheric volume

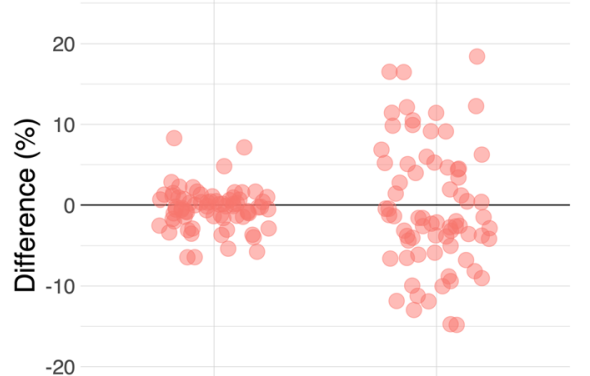

R gray matter $\quad$ R white matter

\section{CERES: lobular volume}

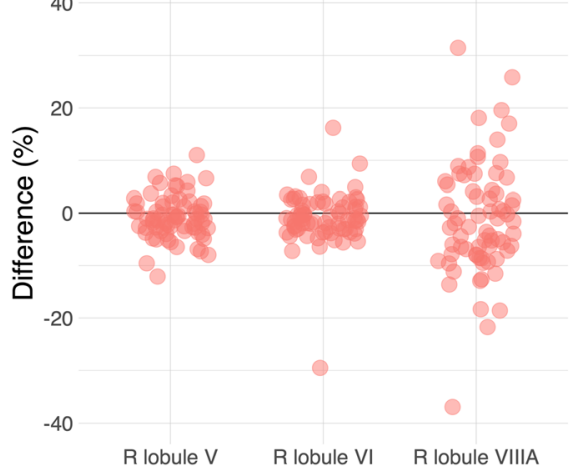

b ACAPULCO: lobular volume

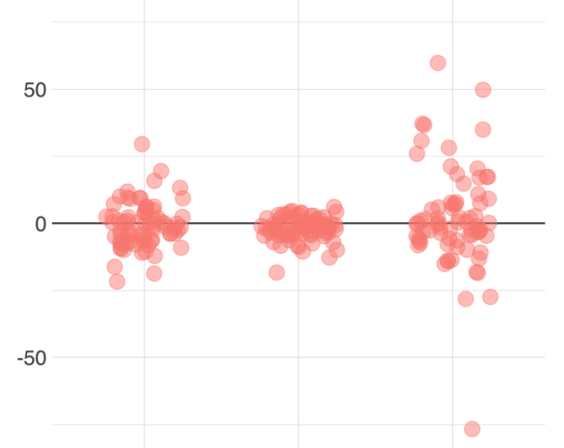

R lobule V R lobule VI R lobule VIIIA e CERES: gray matter volume

40

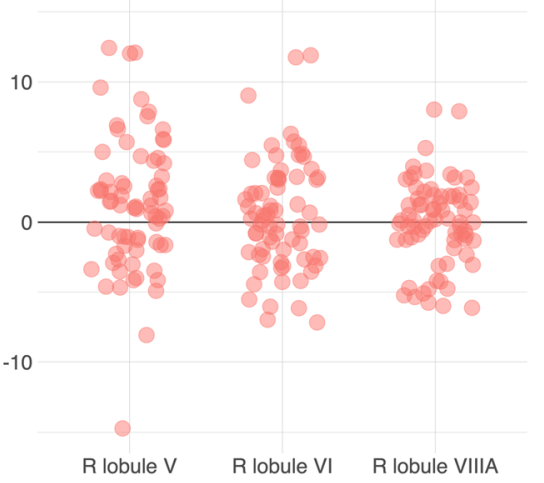

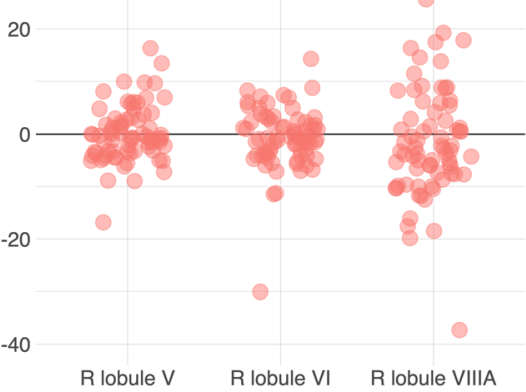

Fig. 6 Long-term reproducibility of cerebellar morphometry in the OASIS-2 study. The graphs show percent difference between the first and second MRI for right gray and white matter using FreeSurfer (a) and the right lobules V, VI, and VIIIA using ACAPULCO (b) and
CERES $(\mathbf{c}, \mathbf{d}, \mathbf{e})$. The mean interval between the two MRIs was $738 \pm$ 249 days (minimum: 182, maximum: 1510 days). Note: the scales of the $y$ axes differ across graphs
Table 2 Image intraclass correlation coefficients (I2C2) and 95\% confidence intervals for cerebellar regions obtained by CERES with data from the Kirby-21 study

\begin{tabular}{lll}
\hline Region & Left & Right \\
\hline Lobules I-II & $0.83(0.79-0.86)$ & $0.83(0.80-0.86)$ \\
Lobule III & $0.87(0.85-0.90)$ & $0.86(0.84-0.88)$ \\
Lobule IV & $0.84(0.82-0.86)$ & $0.85(0.83-0.87)$ \\
Lobule V & $0.83(0.79-0.85)$ & $0.86(0.84-0.88)$ \\
Lobule VI & $0.88(0.85-0.90)$ & $0.89(0.88-0.91)$ \\
Crus I & $0.88(0.87-0.90)$ & $0.89(0.88-0.91)$ \\
Crus II & $0.89(0.87-0.91)$ & $0.89(0.87-0.90)$ \\
Lobule VIIB & $0.87(0.85-0.89)$ & $0.87(0.85-0.89)$ \\
Lobule VIIIA & $0.89(0.87-0.90)$ & $0.89(0.87-0.91)$ \\
Lobule VIIIB & $0.89(0.87-0.91)$ & $0.89(0.87-0.91)$ \\
Lobule IX & $0.89(0.87-0.90)$ & $0.88(0.86-0.90)$ \\
Lobule X & $0.89(0.86-0.90)$ & $0.88(0.85-0.90)$ \\
\hline
\end{tabular}

\section{Discussion}

We present a detailed analysis of the reproducibility of fully automated cerebellar morphometry using three different software packages regarding (1) replicability (two analyses of one data set with identical hardware and software), (2) repeatability (analyses of two data sets taken on the same day), and (3) long-term reproducibility (analyses of two data sets taken months or years apart).

Regarding analysis replicability, we found that the results of FreeSurfer segmentations were identical in all analyses. Replicability was high for CERES parcellations and segmentations in most regions (Fig. 4d-f), although the PatchMatch algorithm employed by CERES is non-deterministic and involves a random search step that is performed iteratively [20]. By contrast, we found substantial differences when performing two identical ACAPULCO analyses of the highresolution ChroPain2 data sets (Fig. 4b). We hypothesized that the submillimeter resolution $(0.75-\mathrm{mm}$ isotropic voxel size) of this data set might have caused problems for ACAPULCO's parcellating network which has been trained 
Table 3 Coefficient of variation (CV) and intraclass correlation coefficient (ICC) for cerebellar regions obtained by FreeSurfer, CERES, and ACAPULCO with data from the Kirby-21 study

\begin{tabular}{|c|c|c|c|c|}
\hline \multirow[t]{2}{*}{ Region } & \multicolumn{2}{|l|}{ Left } & \multicolumn{2}{|l|}{ Right } \\
\hline & $\mathrm{CV}(\%)$ & ICC & $\mathrm{CV}(\%)$ & ICC \\
\hline \multicolumn{5}{|l|}{ FreeSurfer } \\
\hline Gray matter & $1.23(0.95-1.76)$ & $>0.99$ & $1.43(1.10-2.04)$ & $0.99(0.99-1)$ \\
\hline White matter & $3.16(2.43-4.52)$ & $0.96(0.91-0.98)$ & $3.99(3.07-5.70)$ & $0.95(0.90-0.98)$ \\
\hline \multicolumn{5}{|l|}{ CERES } \\
\hline \multicolumn{5}{|c|}{ Lobular volume } \\
\hline Lobules I-II & $12.18(9.37-17.41)$ & $0.93(0.85-0.96)$ & $11.87(9.14-16.97)$ & $0.93(0.86-0.97)$ \\
\hline Lobule III & $2.44(1.87-3.48)$ & $>0.99$ & $3.29(2.53-4.70)$ & $0.97(0.93-0.98)$ \\
\hline Lobule IV & $2.00(1.54-2.86)$ & $0.99(0.97-0.99)$ & $2.11(1.62-3.01)$ & $0.98(0.97-0.99)$ \\
\hline Lobule V & $2.95(2.27-4.21)$ & $0.95(0.89-0.97)$ & $1.51(1.17-2.16)$ & $0.99(0.98-1)$ \\
\hline Lobule VI & $2.01(1.55-2.88)$ & $0.98(0.97-0.99)$ & $0.7(0.54-1.00)$ & $>0.99$ \\
\hline Crus I & $1.94(1.49-2.77)$ & $0.99(0.97-0.99)$ & $1.45(1.12-2.07)$ & $0.99(0.99-1)$ \\
\hline Crus II & $1.61(1.24-2.3)$ & $0.99(0.98-1)$ & $1.68(1.29-2.40)$ & $0.99(0.98-1)$ \\
\hline Lobule VIIB & $2.92(2.25-4.17)$ & $0.98(0.96-0.99)$ & $1.62(1.25-2.32)$ & $0.99(0.99-1)$ \\
\hline Lobule VIIIA & $1.76(1.36-2.52)$ & $0.99(0.98-1)$ & $1.50(1.16-2.15)$ & $0.99(0.99-1)$ \\
\hline Lobule VIIIB & $1.42(1.09-2.02)$ & $0.99(0.99-1)$ & $1.43(1.10-2.04)$ & $>0.99$ \\
\hline Lobule IX & $1.15(0.89-1.65)$ & $>0.99$ & $1.74(1.34-2.49)$ & $0.99(0.99-1)$ \\
\hline Lobule X & $1.94(1.49-2.77)$ & $0.99(0.97-0.99)$ & $1.84(1.41-2.63)$ & $0.98(0.97-0.99)$ \\
\hline \multicolumn{5}{|c|}{ Cortical thickness } \\
\hline Lobules I-II & $11.95(9.19-17.07)$ & $0.80(0.62-0.90)$ & $10.30(7.92-14.71)$ & $0.83(0.68-0.92)$ \\
\hline Lobule III & $4.23(3.26-6.05)$ & $0.91(0.82-0.96)$ & $4.62(3.55-6.60)$ & $0.90(0.81-0.95)$ \\
\hline Lobule IV & $1.6(1.23-2.29)$ & $0.90(0.79-0.95)$ & $1.43(1.10-2.05)$ & $0.94(0.89-0.97)$ \\
\hline Lobule V & $1.02(0.78-1.46)$ & $0.87(0.75-0.94)$ & $1.11(0.85-1.58)$ & $0.94(0.89-0.97)$ \\
\hline Lobule VI & $0.57(0.44-0.81)$ & $0.88(0.76-0.94)$ & $0.58(0.45-0.83)$ & $0.93(0.85-0.96)$ \\
\hline Crus I & $1.22(0.94-1.74)$ & $0.82(0.66-0.91)$ & $1.36(1.04-1.94)$ & $0.86(0.72-0.93)$ \\
\hline Crus II & $1.02(0.78-1.46)$ & $0.82(0.66-0.91)$ & $1.02(0.79-1.46)$ & $0.93(0.85-0.96)$ \\
\hline Lobule VIIB & $0.77(0.59-1.10)$ & $0.89(0.79-0.95)$ & $0.86(0.66-1.23)$ & $0.79(0.60-0.89)$ \\
\hline Lobule VIIIA & $0.66(0.51-0.95)$ & $0.96(0.92-0.98)$ & $1.39(1.07-1.98)$ & $0.77(0.57-0.88)$ \\
\hline Lobule VIIIB & $3.33(2.56-4.76)$ & $0.81(0.64-0.91)$ & $3.39(2.60-4.84)$ & $0.75(0.54-0.87)$ \\
\hline Lobule IX & $5.01(3.85-7.15)$ & $0.84(0.69-0.92)$ & $4.56(3.51-6.51)$ & $0.75(0.54-0.87)$ \\
\hline Lobule X & $6.02(4.63-8.60)$ & $0.80(0.63-0.90)$ & $7.25(5.58-10.37)$ & $0.84(0.70-0.92)$ \\
\hline \multicolumn{5}{|c|}{ Gray matter volume } \\
\hline Lobules I-II & $20.92(16.09-29.89)$ & $0.88(0.77-0.94)$ & $12.98(9.99-18.55)$ & $0.94(0.88-0.97)$ \\
\hline Lobule III & $2.83(2.18-4.05)$ & $0.98(0.95-0.99)$ & $3.72(2.86-5.32)$ & $0.96(0.92-0.98)$ \\
\hline Lobule IV & $1.57(1.21-2.25)$ & $0.99(0.98-1)$ & $1.80(1.39-2.58)$ & $0.99(0.98-0.99)$ \\
\hline Lobule V & $3.52(2.70-5.02)$ & $0.92(0.83-0.96)$ & $1.72(1.32-2.46)$ & $0.99(0.98-0.99)$ \\
\hline Lobule VI & $2.29(1.76-3.27)$ & $0.98(0.96-0.99)$ & $0.72(0.55-1.02)$ & $>0.99$ \\
\hline Crus I & $2.01(1.54-2.87)$ & $0.99(0.97-0.99)$ & $1.62(1.25-2.32)$ & $0.99(0.98-1)$ \\
\hline Crus II & $1.62(1.25-2.32)$ & $0.99(0.98-1)$ & $1.50(1.15-2.14)$ & $0.99(0.98-1)$ \\
\hline Lobule VIIB & $3.04(2.34-4.34)$ & $0.98(0.95-0.99)$ & $1.68(1.29-2.40)$ & $0.99(0.99-1)$ \\
\hline Lobule VIIIA & $1.76(1.36-2.52)$ & $0.99(0.98-1)$ & $1.91(1.47-2.73)$ & $0.99(0.98-1)$ \\
\hline Lobule VIIIB & $2.44(1.88-3.49)$ & $0.98(0.96-0.99)$ & $1.95(1.50-2.78)$ & $0.99(0.98-1)$ \\
\hline Lobule IX & $2.76(2.13-3.95)$ & $0.98(0.95-0.99)$ & $2.56(1.97-3.67)$ & $0.98(0.96-0.99)$ \\
\hline Lobule X & $3.16(2.43-4.52)$ & $0.97(0.93-0.98)$ & $3.10(2.39-4.43)$ & $0.97(0.93-0.98)$ \\
\hline \multicolumn{5}{|l|}{ ACAPULCO } \\
\hline Lobules I-III & $2.90(2.23-4.15)$ & $0.98(0.97-0.99)$ & $3.76(2.89-5.37)$ & $0.95(0.90-0.98)$ \\
\hline
\end{tabular}


Table 3 (continued)

\begin{tabular}{lllll}
\hline Region & Left & & Right \\
\hline Lobule IV & $2.60(2-3.72)$ & $0.95(0.90-0.98)$ & $2.40(1.85-3.43)$ & $0.98(0.96-0.99)$ \\
Lobule V & $3.08(2.37-4.40)$ & $0.95(0.91-0.98)$ & $2.71(2.08-3.87)$ & $0.97(0.94-0.99)$ \\
Lobule VI & $1.26(0.97-1.81)$ & $0.99(0.99-1)$ & $1.08(0.83-1.54)$ & $0.99(0.99-1)$ \\
Lobule VIIAf & $1.16(0.89-1.65)$ & $>0.99$ & $2.79(2.14-3.98)$ & $0.99(0.98-1)$ \\
Lobule VIIAt & $2.11(1.62-3.01)$ & $0.99(0.98-0.99)$ & $3.12(2.40-4.47)$ & $0.98(0.96-0.99)$ \\
Lobule VIIB & $4.76(3.66-6.80)$ & $0.93(0.85-0.96)$ & $3.88(2.98-5.54)$ & $0.96(0.91-0.98)$ \\
Lobule VIIIA & $4.64(3.57-6.63)$ & $0.97(0.93-0.98)$ & $2.28(1.76-3.26)$ & $0.99(0.98-0.99)$ \\
Lobule VIIIB & $3.67(2.83-5.25)$ & $0.98(0.96-0.99)$ & & $0.97(0.95-0.99)$ \\
Lobule IX & $2.13(1.64-3.04)$ & $0.99(0.97-0.99)$ & & $0.99(0.97-0.99)$ \\
Lobule X & $2.04(1.57-2.92)$ & $0.98(0.97-0.99)$ & & $0.96(0.92-0.98)$ \\
Vermis VI & Midline & & & \\
Vermis VII & $1.32(1.02-1.89)$ & $0.99(0.98-1)$ & & \\
Vermis VIII & $2.39(1.84-3.42)$ & $0.98(0.96-0.99)$ & & \\
Vermis IX & $1.62(1.25-2.32)$ & $0.99(0.99-1)$ & & \\
Vermis X & $1.53(1.17-2.18)$ & $0.99(0.98-1)$ & & \\
\hline
\end{tabular}

The table presents the CV and ICC with lower and upper 95\% confidence intervals

with MPRAGE images resampled to 1-mm isotropic resolution [24]. Therefore, we assessed ACAPULCO's analysis replicability with data from the Kirby-21 study $\left(1 \times 1 \times 1.2 \mathrm{~mm}^{3}\right.$ voxel size). Differences between two identical ACAPULCO analyses were lower in the Kirby-21 study compared to the ChroPain 2 study (Fig. 4b-c) but still relatively high, with most differences $< \pm 5 \%$.

Assessment of repeatability revealed a remarkably similar picture for all software packages (Fig. 5). Most differences between the first and the second MRI taken on the same day were < $\pm 5 \%$. This result presents an estimation of the reproducibility with which cerebellar subdivisions can be determined with a recent MRI system at 3 Tesla, a widely used MPRAGE sequence, and a fully automated segmentation and/or parcellation software for individual participants today. For ACAPULCO, intraclass correlation coefficients in our study using the Kirby21 data set (Table 3) were similar to the ICCs reported in Han et al. [24], although both studies used different algorithms.

For comparison, estimation of cerebral cortical thicknesses using FreeSurfer demonstrated an overall higher reproducibility with differences between scans taken within minutes of $\leq \pm$ $1.9 \%$ and between scans taken within weeks of $\leq \pm 2.3 \%$ [56]. Of course, the reported differences between two scans of one person are a complex mixture of several factors, including not only imperfections of the image analysis software used, but also of scanner hardware and MRI sequences, and differences in the positioning of the head. Using a high-resolution sequence (e.g., with a $0.75-\mathrm{mm}$ isotropic voxel size) and/or a higher magnetic field strength (i.e., 7 Tesla) is expected to improve not only assessment of cerebral cortical thicknesses [57] but also of cerebellar volumes and cortical thicknesses due to reduced partial volume effects or increased signal-tonoise-ratios. As the developers of CERES acknowledge, the main limitation of their analysis software is the small library of only five manually labeled cerebellar templates on which CERES relies at present [20]. Hopefully, the developers will include additional templates in future versions of their software, likely improving segmentation and parcellation results.

As expected, long-term reproducibility of cerebellar morphometry was lower than repeatability on the same day. Brain volumes and cortical thicknesses change over time, not only due to aging but also due to factors unrelated to aging, such as diurnal factors [58], hydration [59], or alcohol intake [60]. In single cases, both CERES and ACAPULCO analyses resulted in dramatic differences, suggesting mislabeling of large parts of cerebellar regions.

Limitations of the Study The presented study used only one specific MRI pulse sequence performed on one MRI scanner to assess replicability, repeatability, and long-term reproducibility of cerebellar morphometry. The only exception was the assessment of analysis replicability using ACAPULCO; here, we used two sequences with different voxel dimensions. Our results suggested that ACAPULCO's analysis replicability is reduced in sequences with submillimeter voxel resolution. For future software development and research, we recommend to test a specific analysis pipeline with several different MRI sequences (including different voxel dimensions) performed with different scanner hardware. These tests would be of particular importance for the assessment of long-term reproducibility, because longitudinal studies often use (sometimes slightly) different pulse sequences over time or involve scanner upgrades or replacement. 
Table 4 Coefficient of variation (CV) and intraclass correlation coefficient (ICC) for cerebellar regions obtained by FreeSurfer, CERES, and ACAPULCO with data from the OASIS-2 study

\begin{tabular}{|c|c|c|c|c|}
\hline \multirow[t]{2}{*}{ Region } & \multicolumn{2}{|l|}{ Left } & \multicolumn{2}{|l|}{ Right } \\
\hline & CV $(\%)$ & ICC & $\mathrm{CV}(\%)$ & ICC \\
\hline \multicolumn{5}{|l|}{ FreeSurfer } \\
\hline Gray matter & $2.34(2.01-2.80)$ & $0.98(0.96-0.98)$ & $1.84(1.58-2.20)$ & $0.97(0.96-0.98)$ \\
\hline White matter & $5.21(4.48-6.23)$ & $0.38(0.20-0.53)$ & $5.42(4.66-6.48)$ & $0.42(0.25-0.57)$ \\
\hline \multicolumn{5}{|l|}{ CERES } \\
\hline \multicolumn{5}{|c|}{ Lobular volume } \\
\hline Lobules I-II & $27.34(23.51-32.66)$ & $0.58(0.44-0.70)$ & $15.89(13.66-18.99)$ & $0.73(0.63-0.81)$ \\
\hline Lobule III & 8.15 (7.01-9.74) & $0.84(0.77-0.89)$ & $8.55(7.36-10.22)$ & $0.77(0.68-0.84)$ \\
\hline Lobule IV & $3.87(3.33-4.62)$ & $0.94(0.91-0.96)$ & $4.54(3.91-5.43)$ & $0.94(0.92-0.96)$ \\
\hline Lobule V & $3.17(2.72-3.78)$ & $0.96(0.95-0.97)$ & $2.95(2.53-3.52)$ & $0.95(0.93-0.97)$ \\
\hline Lobule VI & $2.55(2.20-3.05)$ & $0.97(0.96-0.98)$ & $3.37(2.90-4.03)$ & $0.96(0.94-0.97)$ \\
\hline Crus I & $3.04(2.61-3.63)$ & $0.96(0.94-0.97)$ & $3.37(2.90-4.03)$ & $0.96(0.94-0.97)$ \\
\hline Crus II & $4.20(3.61-5.02)$ & $0.95(0.92-0.96)$ & $5.13(4.41-6.13)$ & $0.90(0.85-0.93)$ \\
\hline Lobule VIIB & $6.95(5.98-8.31)$ & $0.89(0.84-0.92)$ & $7.84(6.74-9.36)$ & $0.82(0.74-0.87)$ \\
\hline Lobule VIIIA & $7.15(6.15-8.54)$ & $0.87(0.81-0.91)$ & $7.97(6.85-9.52)$ & $0.84(0.78-0.89)$ \\
\hline Lobule VIIIB & $5.68(4.89-6.79)$ & $0.89(0.84-0.92)$ & $7.68(6.61-9.18)$ & $0.82(0.75-0.88)$ \\
\hline Lobule IX & $4.19(3.60-5.00)$ & $0.96(0.94-0.97)$ & $4.80(4.13-5.73)$ & $0.94(0.92-0.96)$ \\
\hline Lobule X & $5.32(4.57-6.36)$ & $0.88(0.82-0.92)$ & $5.08(4.37-6.07)$ & $0.88(0.83-0.92)$ \\
\hline \multicolumn{5}{|c|}{ Cortical thickness } \\
\hline Lobules I-II & $22.13(19.03-26.45)$ & $0.35(0.16-0.51)$ & $24.73(21.26-29.55)$ & $0.22(0.03-0.40)$ \\
\hline Lobule III & $8.50(7.31-10.15)$ & $0.48(0.32-0.62)$ & $8.85(7.61-10.57)$ & $0.36(0.18-0.52)$ \\
\hline Lobule IV & $3.19(2.75-3.82)$ & $0.52(0.37-0.65)$ & $3.36(2.89-4.02)$ & $0.61(0.47-0.72)$ \\
\hline Lobule V & $2.53(2.18-3.03)$ & $0.67(0.55-0.77)$ & $3.21(2.76-3.84)$ & $0.65(0.52-0.75)$ \\
\hline Lobule VI & $2.75(2.36-3.28)$ & $0.38(0.20-0.53)$ & $2.70(2.33-3.23)$ & $0.48(0.31-0.61)$ \\
\hline Crus I & $3.87(3.33-4.62)$ & $0.71(0.59-0.79)$ & $3.45(2.97-4.12)$ & $0.71(0.60-0.79)$ \\
\hline Crus II & $5.49(4.72-6.56)$ & $0.56(0.41-0.68)$ & $4.39(3.77-5.24)$ & $0.55(0.40-0.67)$ \\
\hline Lobule VIIB & $3.26(2.81-3.90)$ & $0.45(0.28-0.59)$ & $2.13(1.84-2.55)$ & $0.45(0.28-0.59)$ \\
\hline Lobule VIIIA & $1.86(1.60-2.22)$ & $0.58(0.44-0.70)$ & $2.14(1.84-2.56)$ & $0.37(0.19-0.52)$ \\
\hline Lobule VIIIB & $2.12(1.83-2.54)$ & $0.53(0.37-0.66)$ & $2.10(1.81-2.51)$ & $0.48(0.32-0.62)$ \\
\hline Lobule IX & $4.81(4.14-5.75)$ & $0.37(0.19-0.53)$ & $4.66(4.01-5.57)$ & $0.42(0.24-0.56)$ \\
\hline Lobule X & $10.08(8.67-12.05)$ & $0.72(0.61-0.80)$ & $10.15(8.73-12.12)$ & $0.71(0.60-0.80)$ \\
\hline \multicolumn{5}{|c|}{ Gray matter volume } \\
\hline Lobules I-II & $32.49(27.94-38.82)$ & $0.60(0.46-0.71)$ & $22.63(19.46-27.04)$ & $0.54(0.39-0.67)$ \\
\hline Lobule III & $9.39(8.07-11.22)$ & $0.81(0.73-0.87)$ & $8.53(7.33-10.19)$ & $0.79(0.70-0.85)$ \\
\hline Lobule IV & $4.39(3.78-5.25)$ & $0.92(0.89-0.95)$ & $4.83(4.16-5.78)$ & $0.94(0.91-0.96)$ \\
\hline Lobule V & $4.13(3.55-4.93)$ & $0.94(0.91-0.96)$ & $3.64(3.13-4.35)$ & $0.92(0.89-0.95)$ \\
\hline Lobule VI & $2.81(2.41-3.36)$ & $0.97(0.95-0.98)$ & $3.97(3.41-4.74)$ & $0.94(0.91-0.96)$ \\
\hline Crus I & $3.00(2.58-3.58)$ & $0.96(0.95-0.98)$ & $3.00(2.58-3.59)$ & $0.97(0.95-0.98)$ \\
\hline Crus II & $5.17(4.45-6.18)$ & $0.92(0.88-0.94)$ & $5.70(4.90-6.82)$ & $0.87(0.82-0.91)$ \\
\hline Lobule VIIB & $7.48(6.44-8.94)$ & $0.87(0.81-0.91)$ & $8.03(6.91-9.60)$ & $0.80(0.71-0.96)$ \\
\hline Lobule VIIIA & $7.11(6.12-8.50)$ & $0.87(0.81-0.91)$ & $7.66(6.59-9.15)$ & $0.85(0.78-0.90)$ \\
\hline Lobule VIIIB & $6.12(5.26-7.31)$ & $0.87(0.81-0.91)$ & $7.75(6.66-9.26)$ & $0.82(0.75-0.87)$ \\
\hline Lobule IX & $4.04(3.48-4.83)$ & $0.96(0.94-0.97)$ & $4.46(3.83-5.33)$ & $0.95(0.92-0.96)$ \\
\hline Lobule X & $5.03(4.33-6.01)$ & $0.89(0.85-0.93)$ & $5.55(4.78-6.64)$ & $0.87(0.81-0.91)$ \\
\hline \multicolumn{5}{|l|}{ ACAPULCO } \\
\hline Lobules I-III & $12.54(10.79-14.99)$ & $0.74(0.64-0.82)$ & $10.48(9.01-12.52)$ & $0.81(0.74-0.87)$ \\
\hline Lobule IV & 8.17 (7.02-9.76) & $0.81(0.73-0.87)$ & $6.23(5.35-7.44)$ & $0.86(0.80-0.91)$ \\
\hline
\end{tabular}


Table 4 (continued)

\begin{tabular}{|c|c|c|c|c|}
\hline Region & Left & & Right & \\
\hline Lobule V & $7.90(6.80-9.44)$ & $0.80(0.71-0.86)$ & $5.83(5.01-6.96)$ & $0.86(0.80-0.91)$ \\
\hline Lobule VI & $4.19(3.60-5.00)$ & $0.93(0.90-0.95)$ & $3.48(2.99-4.16)$ & $0.95(0.93-0.97)$ \\
\hline Lobule VIIAf & $2.62(2.25-3.13)$ & $0.97(0.96-0.98)$ & $2.84(2.44-3.39)$ & $0.96(0.95-0.98)$ \\
\hline Lobule VIIAt & $7.38(6.34-8.82)$ & $0.81(0.74-0.87)$ & $7.84(6.75-9.37)$ & $0.82(0.75-0.88)$ \\
\hline Lobule VIIB & $8.41(7.23-10.05)$ & $0.80(0.72-0.86)$ & $11.70(10.06-13.98)$ & $0.69(0.58-0.78)$ \\
\hline Lobule VIIIA & $8.85(7.61-10.57)$ & $0.81(0.73-0.87)$ & $11.28(9.70-13.48)$ & $0.78(0.69-0.84)$ \\
\hline Lobule VIIIB & $11.32(9.73-13.52)$ & $0.81(0.73-0.87)$ & $10.56(9.08-12.62)$ & $0.74(0.63-0.81)$ \\
\hline Lobule IX & $5.02(4.31-5.99)$ & $0.93(0.91-0.96)$ & $5.86(5.04-7.00)$ & $0.91(0.87-0.94)$ \\
\hline \multirow[t]{2}{*}{ Lobule X } & $9.26(7.97-11.07)$ & $0.74(0.63-0.81)$ & $8.60(7.40-10.28)$ & $0.70(0.59-0.79)$ \\
\hline & Midline & & & \\
\hline Vermis VI & $6.83(5.87-8.16)$ & $0.81(0.73-0.87)$ & & \\
\hline Vermis VII & $39.77(34.20-47.52)$ & $0.11(-0.09-0.29)^{*}$ & & \\
\hline Vermis VIII & $3.39(2.92-4.05)$ & $0.95(0.92-0.96)$ & & \\
\hline Vermis IX & $4.93(4.24-5.89)$ & $0.90(0.85-0.93)$ & & \\
\hline Vermis $\mathrm{X}$ & $8.11(6.97-9.69)$ & $0.76(0.66-0.83)$ & & \\
\hline
\end{tabular}

The table presents the CV and ICC with lower and upper 95\% confidence intervals

*Calculation of the ICC for Vermis VII (ACAPULCO) includes one outlier (median: $1.35 \mathrm{~cm}^{3}$, outlier: $7.94 \mathrm{~cm}^{3}$ ). Without the outlier, the ICC is $0.83(0.76-0.88$ )

\section{Recommendations for Use of Automated Cerebellar Morphometry}

Based on the presented analyses, we recommend the following steps to improve the design, data analysis, and interpretation of future neuroimaging studies:

1. Quality control through visual inspection of all labeled regions. Corroborating the results of Kavaklioglu et al. [61], we recognized that FreeSurfer frequently mislabeled voxels representing the dura mater or the dural sinuses as cerebellar gray matter. The number of these voxels is usually small compared to the entire gray matter of the left or right cerebellum. Manual correction of labels and recomputing of cerebellar volumes is possible, but would require substantial expertise and time [62], and is therefore not feasible in large-scale studies. Of note, the locating network used in ACAPULCO failed in one analysis. In this case, the parcellating network mislabeled all voxels and finished without error message. Thus, we strongly recommend the visual inspection of all results of neuroimaging pipelines, including automated cerebellar morphometry. Visual inspection of subcortical FreeSurfer results requires manual loading of .mgz files in FreeSurfer's Freeview file viewer or in another viewer capable of displaying .mgz files (e.g., FSLeyes). Visual inspection of CERES and ACAPULCO results is less timeconsuming because both analysis packages create report pages in pdf or html format for convenient inspection.

2. Assessment of analysis replicability. Many MRI analysis packages include stochastic algorithms, such as random seed generation for the initialization of analyses [63]. Given the remarkable differences found in identical analyses by ACAPULCO, we recommend reporting the analysis replicability for every neuroimaging pipeline, including cerebellar morphometry.

3. Assessment of repeatability. For cross-sectional studies, we recommend reporting the repeatability of the selected neuroimaging pipeline in addition to its analysis replicability. The data set for assessment of repeatability should include two identical MRI scans taken on the same day, ideally directly one after another, but with repositioning in between, to minimize true changes in brain volumes or cortical thicknesses.

4. Assessment of long-term reproducibility. For the design of a longitudinal study, we recommend investigating the long-term reproducibility of the selected neuroimaging pipeline in addition to its analysis replicability. The data set for estimation of long-term reproducibility should include two or more scans, taken in time intervals comparable to the planned longitudinal study. The obtained results should guide the decision if the expected changes may be observed with the sample size and the study design under consideration [64].

\section{Conclusions}

Based on its high accuracy [19], its overall high reproducibility shown here, and its ability to differentiate between entire lobular volumes, gray matter lobular volumes, and lobular cortical thicknesses, CERES is a powerful tool to investigate 
cerebellar morphometry. Cerebellar morphometry is expected to provide important biomarkers for cerebellar aging and disease. Reliable neuroimaging biomarkers depend on reproducible analyses. For every neuroimaging pipeline, not only for cerebellar morphometry, reproducibility should be investigated, reported, and utilized for the interpretation of its results.

Acknowledgments The authors wish to thank Stefan Harfst and Fynn Schwietzer, Scientific Computing, University of Oldenburg, Germany, for continuous support with the HPC cluster.

Authors' Contributions All authors contributed to the study conception and design. Material preparation and data collection were performed by Peter Sörös and Louise Wölk. Data analysis was performed by Peter Sörös and Frank Klawonn. The ChroPain2 study was conceived by Carsten Bantel and Peter Sörös. The first draft of the manuscript was written by Peter Sörös, and all authors commented on previous versions of the manuscript. All authors read and approved the final manuscript.

Funding Open Access funding enabled and organized by Projekt DEAL. The ChroPain2 study was supported by the Neuroimaging Unit, University of Oldenburg, funded by grants from the German Research Foundation (DFG; 3 T MRI INST 184/152-1 FUGG and MEG INST 184/ 148-1 FUGG). The high-performance computer cluster CARL, University of Oldenburg, is funded by a grant from the German Research Foundation (DFG; INST 184/157-1 FUGG).

\section{Compliance with Ethical Standards}

Conflicts of Interests The authors declare that they have no conflicts of interest.

Ethics Approval This study was performed in line with the principles of the Declaration of Helsinki. Approval for the ChroPain2 study was granted by the Medical Research Ethics Board, University of Oldenburg, Germany (2017-059). Approval for the Kirby-21 and the OASIS-2 studies were granted by local Research Ethics Boards.

Consent to Participate Informed consent was obtained from all individual participants included in the study.

Open Access This article is licensed under a Creative Commons Attribution 4.0 International License, which permits use, sharing, adaptation, distribution and reproduction in any medium or format, as long as you give appropriate credit to the original author(s) and the source, provide a link to the Creative Commons licence, and indicate if changes were made. The images or other third party material in this article are included in the article's Creative Commons licence, unless indicated otherwise in a credit line to the material. If material is not included in the article's Creative Commons licence and your intended use is not permitted by statutory regulation or exceeds the permitted use, you will need to obtain permission directly from the copyright holder. To view a copy of this licence, visit http://creativecommons.org/licenses/by/4.0/.

\section{References}

1. Ahmadian N, van Baarsen K, van Zandvoort M, Robe PA. The cerebellar cognitive affective syndrome - a meta-analysis. Cerebellum. 2019;18(5):941-50.
2. Guell X, Schmahmann J. Cerebellar functional anatomy: a didactic summary based on human fMRI evidence. Cerebellum. 2020;19(1):1-5.

3. Argyropoulos GPD, van Dun K, Adamaszek M, Leggio M, Manto M, Masciullo M, et al. The cerebellar cognitive affective/ Schmahmann syndrome: a task force paper. Cerebellum. 2020;19(1):102-25.

4. Rolando L. Saggio sopra la vera struttura del cervello dell'uomo e degl' animali e sopra le funzioni del sistema nervoso. Privilegiata, Sassari: Stamperìa da S.S.R.M; 1809.

5. Flourens P. Recherches expérimentales sur les propriétés et les fonctions du système nerveux, dans les animaux vertébrés. Paris: Crevot; 1824.

6. Thach WT. A role for the cerebellum in learning movement coordination. Neurobiol Learn Mem. 1998;70(1-2):177-88.

7. Imamizu H, Miyauchi S, Tamada T, Sasaki Y, Takino R, Pütz B, et al. Human cerebellar activity reflecting an acquired internal model of a new tool. Nature. 2000;403(6766):192-5.

8. Schmahmann JD, Sherman JC. The cerebellar cognitive affective syndrome. Brain. 1998;121(Pt 4):561-79.

9. Schmahmann JD. The cerebellum and cognition. Neurosci Lett. 2019;688:62-75.

10. Adamaszek M, D’Agata F, Ferrucci R, Habas C, Keulen S, Kirkby $\mathrm{KC}$, et al. Consensus paper: cerebellum and emotion. Cerebellum. 2017;16(2):552-76.

11. D'Angelo E, De Zeeuw CI. Timing and plasticity in the cerebellum: focus on the granular layer. Trends Neurosci. 2009;32(1):30-40.

12. Bareš M, Apps R, Avanzino L, Breska A, D’Angelo E, Filip P, et al. Consensus paper: decoding the contributions of the cerebellum as a time machine. From neurons to clinical applications. Cerebellum. 2019;18(2):266-86.

13. Andersen BB, Gundersen HJ, Pakkenberg B. Aging of the human cerebellum: a stereological study. J Comp Neurol. 2003;466(3): 356-65.

14. Gellersen HM, Guo CC, O'Callaghan C, Tan RH, Sami S, Hornberger M. Cerebellar atrophy in neurodegeneration - a metaanalysis. J Neurol Neurosurg Psychiatry. 2017;88(9):780-8.

15. Kansal K, Yang Z, Fishman AM, Sair HI, Ying SH, Jedynak BM, et al. Structural cerebellar correlates of cognitive and motor dysfunctions in cerebellar degeneration. Brain. 2017;140(3):707-20.

16. Diedrichsen J. A spatially unbiased atlas template of the human cerebellum. Neuroimage. 2006;33(1):127-38.

17. Steele CJ, Chakravarty MM. Gray-matter structural variability in the human cerebellum: lobule-specific differences across sex and hemisphere. Neuroimage. 2018;170:164-73.

18. Park MT, Pipitone J, Baer LH, Winterburn JL, Shah Y, Chavez S, et al. Derivation of high-resolution MRI atlases of the human cerebellum at $3 \mathrm{~T}$ and segmentation using multiple automatically generated templates. Neuroimage. 2014;95:217-31.

19. Carass A, Cuzzocreo JL, Han S, Hernandez-Castillo CR, Rasser PE, Ganz M, et al. Neuroimage. 2018;183:150-72.

20. Romero JE, Coupé P, Giraud R, Ta VT, Fonov V, Park MTM, et al. Ceres: a new cerebellum lobule segmentation method. Neuroimage. 2017;147:916-24.

21. Diedrichsen J, Balsters JH, Flavell J, Cussans E, Ramnani N. A probabilistic MR atlas of the human cerebellum. Neuroimage. 2009;46(1):39-46.

22. Nichols TE, Das S, Eickhoff SB, Evans AC, Glatard T, Hanke M, et al. Best practices in data analysis and sharing in neuroimaging using MRI. Nat Neurosci. 2017;20(3):299-303.

23. Fischl B, Salat DH, Busa E, Albert M, Dieterich M, Haselgrove C, et al. Whole brain segmentation: automated labeling of neuroanatomical structures in the human brain. Neuron. 2002;33(3):341-55.

24. Han S, Carass A, He Y, Prince JL. Automatic cerebellum anatomical parcellation using U-Net with locally constrained optimization. Neuroimage. 2020;218:116819. 
25. Sörös P, Bantel C. Chronic noncancer pain is not associated with accelerated brain aging as assessed by structural magnetic resonance imaging in patients treated in specialized outpatient clinics. Pain. 2020;161(3):641-50.

26. Mugler JP, Brookeman JR. Three-dimensional magnetization-prepared rapid gradient echo imaging (3D MP RAGE). Magn Reson Med. 1990;15(1):152-7.

27. Hamilton J, Franson D, Seiberlich N. Recent advances in parallel imaging for MRI. Prog Nucl Magn Reson Spectrosc. 2017;101:7195.

28. Landman BA, Huang AJ, Gifford A, Vikram DS, Lim IA, Farrell JA, et al. Multi-parametric neuroimaging reproducibility: a 3-T resource study. Neuroimage. 2011;54(4):2854-66.

29. Palumbo L, Bosco P, Fantacci ME, Ferrari E, Oliva P, Spera G, et al. Evaluation of the intra- and inter-method agreement of brain MRI segmentation software packages: a comparison between SPM12 and FreeSurfer v6.0. Phys Med. 2019;64:261-72.

30. Rezende TJR, Campos BM, Hsu J, Li Y, Ceritoglu C, Kutten K, et al. Test-retest reproducibility of a multi-atlas automated segmentation tool on multimodality brain MRI. Brain Behav. 2019;9(10): e01363.

31. Marcus DS, Fotenos AF, Csernansky JG, Morris JC, Buckner RL. Open access series of imaging studies: longitudinal MRI data in nondemented and demented older adults. J Cogn Neurosci. 2010;22(12):2677-84.

32. Hughes CP, Berg L, Danziger WL, Coben LA, Martin RL. A new clinical scale for the staging of dementia. Br J Psychiatry. 1982;140: 566-72.

33. Manjón JV, Coupé P. volBrain: an online MRI brain volumetry system. Front Neuroinform. 2016;10:30.

34. Schmahmann JD, Doyon J, McDonald D, Holmes C, Lavoie K, Hurwitz AS, et al. Three-dimensional MRI atlas of the human cerebellum in proportional stereotaxic space. Neuroimage. 1999;10(3 Pt 1):233-60.

35. Federative International Programme on Anatomical Terminologies. Terminologia Anatomica. Thieme, Stuttgart, Germany; 2011.

36. Fischl B. Freesurfer. Neuroimage. 2012;62(2):774-81.

37. Ségonne F, Dale AM, Busa E, Glessner M, Salat D, Hahn HK, et al. A hybrid approach to the skull stripping problem in MRI. Neuroimage. 2004;22(3):1060-75.

38. Giraud R, Ta VT, Papadakis N, Manjón JV, Collins DL, Coupé P, et al. An optimized PatchMatch for multi-scale and multi-feature label fusion. Neuroimage. 2016;124(Pt A):770-82.

39. Manjón JV, Coupé P, Martí-Bonmatí L, Collins DL, Robles M. Adaptive non-local means denoising of MR images with spatially varying noise levels. J Magn Reson Imaging. 2010;31(1):192-203.

40. Tustison NJ, Avants BB, Cook PA, Zheng Y, Egan A, Yushkevich PA, et al. N4ITK: improved N3 bias correction. IEEE Trans Med Imaging. 2010;29(6):1310.

41. Avants BB, Tustison NJ, Song G, Cook PA, Klein A, Gee JC. A reproducible evaluation of ANTs similarity metric performance in brain image registration. Neuroimage. 2011;54(3):2033-44.

42. Tustison NJ, Cook PA, Klein A, Song G, Das SR, Duda JT, et al. Large-scale evaluation of ANTs and FreeSurfer cortical thickness measurements. Neuroimage. 2014;99:166-79.

43. Coupé P, Manjón JV, Fonov V, Pruessner J, Robles M, Collins DL. Patch-based segmentation using expert priors: application to hippocampus and ventricle segmentation. Neuroimage. 2011;54(2):94054.

44. Ta VT, Giraud R, Collins DL, Coupé P. Optimized PatchMatch for near real time and accurate label fusion. International Conference on Medical Image Computing and ComputerAssisted Intervention, pages 105-112, 2014.
45. Iglesias JE, Liu CY, Thompson PM, Tu Z. Robust brain extraction across datasets and comparison with publicly available methods. IEEE Trans Med Imaging. 2011;30(9):1617-34.

46. Tange O. GNU Parallel. 2018. https://doi.org/10.5281/zenodo. 1146014

47. Shou H, Eloyan A, Lee S, Zipunnikov V, Crainiceanu AN, Nebel $\mathrm{NB}$, et al. Quantifying the reliability of image replication studies: the image intraclass correlation coefficient (I2C2). Cogn Affect Behav Neurosci. 2013;13(4):714-24.

48. R Core Team. R: a language and environment for statistical computing. Vienna, Austria: R Foundation for Statistical Computing; 2020.

49. Revelle W. psych: Procedures for psychological, psychometric, and personality research. Evanston: Northwestern University; 2019.

50. Liljequist D, Elfving B, Roaldsen KS. Intraclass correlation - a discussion and demonstration of basic features. PLoS One. 2019;14(7):e0219854.

51. Shrout PE, Fleiss JL. Intraclass correlations: uses in assessing rater reliability. Psychol Bull. 1979;86(2):420-8.

52. McGraw KO, Wong SP. Forming inferences about some intraclass correlation coefficients. Psychol Methods. 1996;1:30-46.

53. Koo TK, Li MY. A guideline of selecting and reporting intraclass correlation coefficients for reliability research. J Chiropr Med. 2016;15(2):155-63.

54. Muschelli J, Gherman A, Fortin J-P, Avants B, Whitcher B, Clayden JD, et al. Neuroconductor: an R platform for medical imaging analysis. Biostatistics. 2019;20:218-39.

55. Stoodley CJ, Schmahmann JD. Functional topography in the human cerebellum: a meta-analysis of neuroimaging studies. Neuroimage. 2009;44(2):489-501.

56. Wang X, Bauer W, Chiaia N, Dennis M, Gerken M, Hummel J, et al. Longitudinal MRI evaluations of human global cortical thickness over minutes to weeks. Neurosci Lett. 2008;441(2):145-8.

57. Zaretskaya N, Fischl B, Reuter M, Renvall V, Polimeni JR. Advantages of cortical surface reconstruction using submillimeter 7 T MEMPRAGE. Neuroimage. 2018;165:11-26.

58. Nakamura K, Brown RA, Narayanan S, Collins DL, Arnold DL, Alzheimer's Disease Neuroimaging Initiative. Diurnal fluctuations in brain volume: statistical analyses of MRI from large populations. Neuroimage. 2015;118:126-32.

59. Nakamura K, Brown RA, Araujo D, Narayanan S, Arnold DL. Correlation between brain volume change and $\mathrm{T} 2$ relaxation time induced by dehydration and rehydration: implications for monitoring atrophy in clinical studies. Neuroimage Clin. 2014;6:166-70.

60. Geibprasert S, Gallucci M, Krings T. Alcohol-induced changes in the brain as assessed by MRI and CT. Eur Radiol. 2010;20(6): 1492-501.

61. Kavaklioglu T, Guadalupe T, Zwiers M, Marquand AF, Onnink M, Shumskaya E, et al. Structural asymmetries of the human cerebellum in relation to cerebral cortical asymmetries and handedness. Brain Struct Funct. 2017;222(4):1611-23.

62. Wang JY, Ngo MM, Hessl D, Hagerman RJ, Rivera SM. Robust machine learning based correction on automatic segmentation of the cerebellum and brainstem. PLoS One. 2016;11(5):e0156123.

63. Glatard T, Lewis LB, Ferreira da Silva R, Adalat R, Beck N, Lepage $\mathrm{C}$, et al. Reproducibility of neuroimaging analyses across operating systems. Front Neuroinform. 2015;9:12.

64. Velasco-Annis C, Akhondi-Asl A, Stamm A, Warfield SK. Reproducibility of brain MRI segmentation algorithms: empirical comparison of local MAP PSTAPLE, FreeSurfer, and FSL-FIRST. J Neuroimaging. 2018;28(2):162-72.

Publisher's Note Springer Nature remains neutral with regard to jurisdictional claims in published maps and institutional affiliations. 